\title{
Primary Care Interventions to Address COVID-19 Vaccine Hesitancy Among Israel Defense Forces Soldiers
}

\author{
Tomer Talmy $^{1}\left[\right.$ $\cdot$ Bar Cohen $^{1} \cdot$ Itay Nitzan $^{1} \cdot$ Yossi Ben Michael ${ }^{1,2}$ \\ Accepted: 7 May 2021 / Published online: 14 May 2021 \\ (c) The Author(s), under exclusive licence to Springer Science+Business Media, LLC, part of Springer Nature 2021
}

\begin{abstract}
Vaccine hesitancy is an obstacle to achieving high vaccination rates for COVID-19. Current knowledge on vaccine uptake is mostly based on hypothetical intention to vaccinate surveys. We compared intention to vaccinate and real-world vaccine uptake among 511 soldiers in a military unit during an unrestricted, on-site COVID-19 vaccine rollout. Soldiers were offered group lectures, on-site consultations and primary care office visits, discussing concerns on vaccination with a primary care physician. Overall, 359 (70.3\%) soldiers participated in the group lectures, $33(6.5 \%)$ in on-site consultations and $19(3.7 \%)$ attended primary care visits. Overall, 459 (89.8\%) of 511 soldiers vaccinated for COVID-19. Of the 90 soldiers initially refusing, $38(42.2 \%)$ had agreed to receive a vaccine. On-site COVID-19 vaccine rollout joined with primary care communication interventions may maximize vaccine uptake within a young-adult community. Future studies should evaluate the effectiveness of these efforts across different populations in a controlled and comparative manner.
\end{abstract}

Keywords COVID-19 $\cdot$ SARS-CoV-2 $\cdot$ Vaccine $\cdot$ Military $\cdot$ Primary Care $\cdot$ Hesitancy

\section{Introduction}

Widespread public acceptance and uptake of COVID-19 vaccinations is vital to reducing disease burden, both worldwide and on a community level. With authorized vaccines becoming more available, public willingness to vaccinate stands as a key obstacle to achieving a high vaccination rate $[1,2]$. While national and regional approaches to increase vaccine acceptance are essential, primary care physicians are destined to play a major role in engaging and informing

Tomer Talmy

ttalmy@gmail.com

Bar Cohen

barc933@gmail.com

Itay Nitzan

itayn30@gmail.com

Yossi Ben Michael

yossi10264@gmail.com

1 Israel Defense Forces, Medical Corps, Tel Hashomer, Ramat Gan, Israel

2 Institute for Research in Military Medicine, Faculty of Medicine, The Hebrew University of Jerusalem, Jerusalem, Israel their communities with the aim of gaining public trust and uptake of the COVID-19 vaccines [3, 4].

The large-majority of data concerning COVID-19 vaccine uptake has been aggregated from hypothetical vaccine uptake surveys $[3,5,6]$ and while rollout of vaccines has begun worldwide, data on vaccine uptake among different populations remains limited. The initial rollout of COVID19 vaccines in Israel was characterized by a relatively high vaccine uptake and efficiency in vaccine administration. However, despite incentivizing measures such as the vaccine passport utilized in Israel [7], hesitancy remains a concern with only $62.4 \%$ of the Israeli population being vaccinated as of April 30, 2021 (Fig. 1) [8]. Vaccine hesitancy seems to be more prevalent in the young adult population, as this population may attribute a lesser risk to COVID-19 [9]. In January 2021, the Israel Defense Forces (IDF) began on-site vaccine rollout within certain units, aiming to vaccinate the entire soldier population in these units, without prespecified prioritization or limit of vaccine doses. Considering the concerns on vaccine acceptance in the young adult military population, we implemented a multi-layered primary care intervention within an IDF unit, with the aim of addressing soldiers' specific concerns regarding COVID-19 vaccination.

The goal of this study was to assess real-world vaccine uptake in a closed setting with high access to COVID-19 


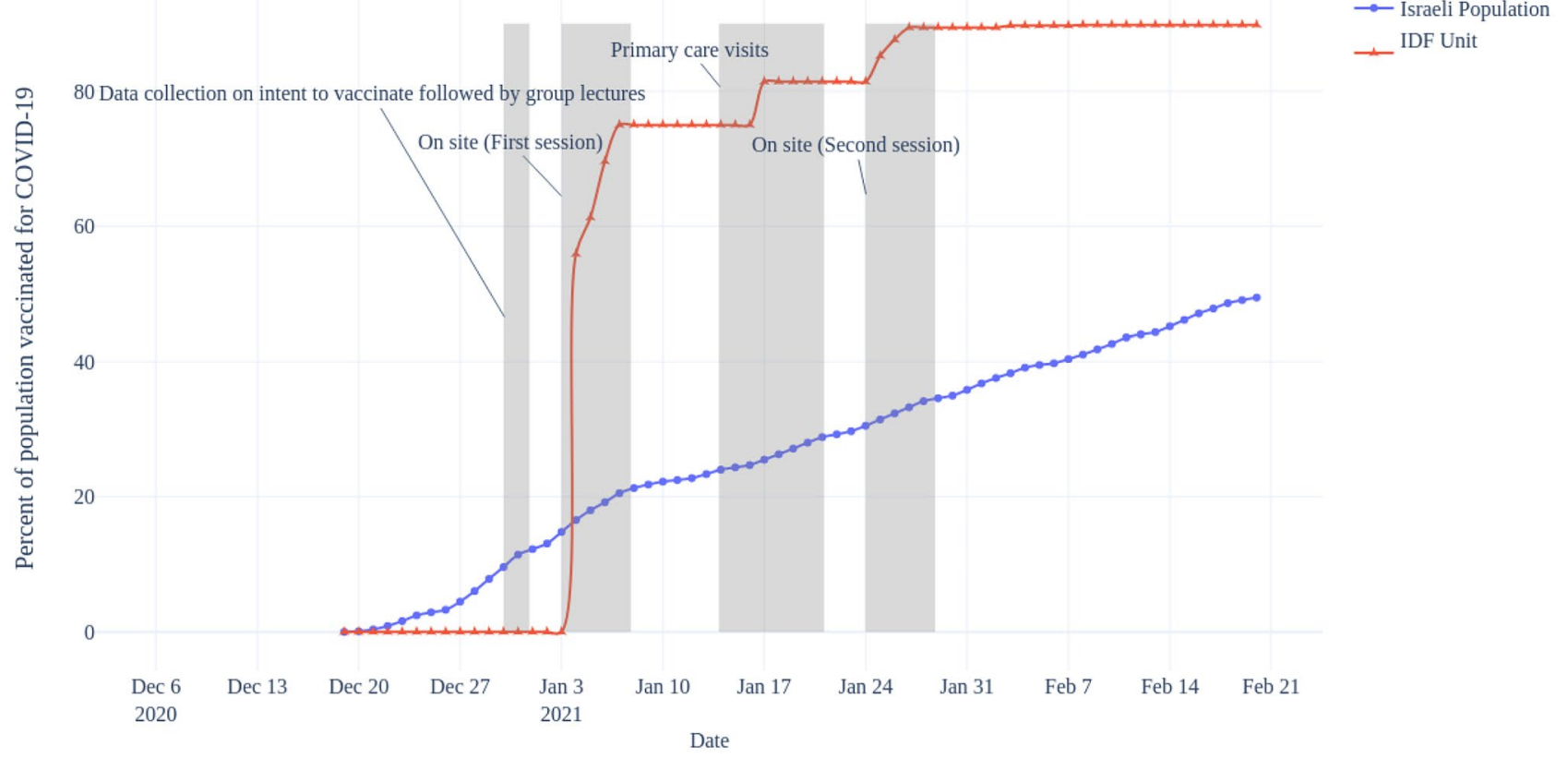

Fig. 1 Percent of population vaccinated for COVID-19 among soldiers in an IDF unit and the entire Israeli population. Data on vaccination in Israel accessed from Our World in Data [8]

vaccines, joined with primary care communication efforts including group lectures, on-site consultations and primary care office visits. We therefore present data on the dynamics of intention to vaccinate versus real-world COVID-19 vaccination and discuss the potential impacts of a highly accessible vaccine rollout on vaccination in this closed soldier community.

\section{Materials and Methods}

This study was conducted as part of a concerted effort to increase vaccination rates within IDF units receiving an allotment of the Pfizer-BioNTech BNT162b2 COVID-19 vaccine [10]. We previously conducted an anonymous online survey during December, 2020, within the same IDF unit, indicating that $62.6 \%$ of 318 survey respondents intended to vaccinate, had one become available, with the rest specifying reasons for hesitancy or concerns regarding a COVID-19 vaccine [9]. The main causes for concern identified in the survey included the safety profile of the mRNA vaccines, rapid development and efficacy.

On December 30, 2020, the unit was included within the IDF's mass vaccination program, allotting it a precise number of BNT162b2 vaccine doses to satisfy the entire demand within the unit. In accordance with this policy, we collected data on intention to vaccinate between December 30 and 31,
2020, prior to vaccine arrival. Calls regarding vaccine intention were made by the unit's secretary and human resources staff without direct involvement of medical staff. The sole intention of these calls was to collect accurate data on intent to vaccinate for purposes of vaccine site logistic arrangements and no informative efforts were made at this juncture. We then implemented several primary care interventions so as to better inform soldiers regarding the COVID-19 vaccine (Fig. 1).

\section{Study Population and Setting}

The study population consisted of all soldiers serving in an IDF unit prior to vaccine rollout. Soldiers who had previously tested positive or had a contraindication for vaccination were excluded from our analysis. For all others, vaccination was entirely voluntary and freely available, without any associated reward or material benefits.

\section{Vaccine Administration}

Soldiers agreeing to vaccinate were exclusively administered the Pfizer-BioNTech BNT162b2 mRNA COVID-19 vaccine. The large-majority of soldiers in this study were vaccinated in a COVID-19 vaccination site constructed 
on the premises of the unit's base, ensuring close proximity and maximum availability. The vaccination site operated daily during two different sessions, the first between January 3 and 7, 2021 and the second between January 24 and 28, 2021 with the aim of allowing for both doses to be received without need for travel or arrangement by the unit's soldiers. A small proportion of soldiers $(n=49$, 9.5\%), unable to complete the two-dose regimen at this vaccination site were referred to other vaccine sites. Doses were administered 21 days apart except in rare cases of soldiers on combat missions or self-quarantine due to close contact exposure.

\section{Primary Care Interventions}

We used three communication approaches to inform soldiers and potentially increase COVID-19 vaccine uptake:

(1) Frontal group lectures Each platoon in the unit attended a non-mandatory 45-min frontal lecture given by the unit's primary care physician. The lecture was administered immediately after collection of data on intention to vaccinate for each platoon. The lecture presented the Phase III trial data of the BNT162b2 vaccine [10] including efficacy, study population, side effects and limitations. Specific slides addressed the concerns inferred in the initial survey administered to soldiers prior to vaccine availability [9], particularly the vaccine's development, safety profile and long-term side effects of previously authorized vaccines. Each session also included a discussion on currently circulating disinformation, potential benefits of a "vaccine passport", and a 15-min Q\&A session.

(2) On-site Consultation Soldiers refusing to or unsure regarding vaccination upon initial questioning were encouraged by their commanders to arrive for physician consultation at the vaccination site during their respective platoon's time-slot. Soldiers who intended to receive a vaccine but had specific concerns were also allowed to privately consult the unit's primary care physician who remained on-site throughout the site's operating times.

(3) Primary care office visits Soldiers who refused vaccination following the initial rollout of the first dose between January 3 and 7, 2021, were contacted to set voluntary appointments for clinic visits to discuss their specific concerns on vaccination in a confidential and discrete manner. All visits were conducted by the unit's primary care physician, and addressed soldiers concern using the current data on the global vaccine rollout and the currently published literature concerning the COVID-19 mRNA vaccines.

\section{Data Collection and Measurements}

Demographics of the study population including sex and age were collected from the IDF's electronic health record (EHR) - CPR $囚$. Data on intention to vaccinate were collected via phone calls made to all soldiers in the unit on December 30-31, 2020, with the aim of issuing vaccine supply demands and identifying soldiers whom were unsure or refused to vaccinate. We collected data on attendance of the frontal lecture sessions and other aforementioned interventions. Real-world vaccination data was recorded between January 1 and February 18, 2021 by means of on-site registration or extracted from the EHR for soldiers vaccinated at remote sites. The data were recorded and anonymized prior to undergoing statistical analysis.

\section{Statistical Analysis}

The demographic characteristics of the study population are presented as means and standard deviations for continuous variables and frequencies for categorical variables. Student's t-test and Fisher's exact test of independence were used to compare differences in continuous and categorical variables, respectively. We calculated a Kaplan-Meier curve to deduce the cumulative vaccination rates during the study's timeframe. Statistical significance was defined as a $p<0.05$ for all tests. SPSS 25.0 software (Chicago, Illinois) was used for all data analyses.

\section{Results}

\section{Demographics}

A total of 511 soldiers were included in the study, 325 (63.6\%) males, $186(36.4 \%)$ females with a mean age of 21.5 years $( \pm 3.6) .293$ soldiers $(57.3 \%)$ were from combat platoons and $186(42.75 \%)$ were from non-combat platoons (Table 1).

\section{Intent to Vaccinate}

Upon initial telephone questioning 397 (77.7\%) soldiers stated they intend to vaccinate, $90(17.6 \%)$ replied that they do not intend to vaccinate and $24(4.7 \%)$ were unsure (Table 1).

\section{Participation in Primary Care Interventions}

Of the 511 soldiers included in the study, 359 (70.3\%) attended a group lecture, 33 (6.5\%) arrived for on-site physician consultation and $19(3.7 \%)$ attended primary care clinic visits. Of the 90 soldiers who stated upon questioning 
Table 1 Demographic characteristics of the study population stratified according to vaccination status

\begin{tabular}{|c|c|c|c|c|}
\hline \multirow[t]{2}{*}{ Characteristics } & \multicolumn{4}{|c|}{ COVID-19 vaccination status } \\
\hline & Vaccinated $(\mathrm{N}=459)$ & Unvaccinated $(\mathrm{N}=52)$ & Total $(\mathrm{N}=511)$ & $p$-value ${ }^{\mathrm{a}}$ \\
\hline \multicolumn{5}{|c|}{ Age—mean $( \pm S D)$, years } \\
\hline & $21.5( \pm 3.6)$ & $20.8( \pm 1.9)$ & $21.5( \pm 3.5)$ & 0.113 \\
\hline \multicolumn{5}{|l|}{$\operatorname{Sex}-\mathrm{N}(\%)$} \\
\hline Male & $294(64.1 \%)$ & $31(59.6 \%)$ & $325(63.6 \%)$ & \multirow[t]{2}{*}{0.313} \\
\hline Female & $165(35.9 \%)$ & $21(40.4 \%)$ & $186(36.4 \%)$ & \\
\hline \multicolumn{5}{|c|}{ Intention to vaccinate $-\mathrm{N}(\%)$} \\
\hline Yes & $397(86.5 \%)$ & $0(0.0 \%)$ & $397(77.7 \%)$ & \multirow[t]{3}{*}{$<0.001^{*}$} \\
\hline No & $38(8.3 \%)$ & $52(100.0 \%)$ & $90(17.6 \%)$ & \\
\hline Unsure & $24(5.2 \%)$ & $0(0.0 \%)$ & $24(4.7 \%)$ & \\
\hline \multicolumn{5}{|c|}{ Attendance of interventions $-\mathrm{N}(\%)$} \\
\hline Group lecture & $331(72.1 \%)$ & $28(53.8 \%)$ & $359(70.3 \%)$ & \multirow[t]{3}{*}{-} \\
\hline On-site consultation & $23(5.0 \%)$ & $10(19.2 \%)$ & $33(6.5 \%)$ & \\
\hline Office-visit & $9(2.0 \%)$ & $10(19.2 \%)$ & $19(3.7 \%)$ & \\
\hline \multicolumn{5}{|l|}{ Platoon-N (\%) } \\
\hline Combat & $282(61.4 \%)$ & $11(21.2 \%)$ & $293(57.3 \%)$ & \multirow[t]{2}{*}{$<0.001^{*}$} \\
\hline Non-combat & $177(38.6 \%)$ & $41(78.8 \%)$ & $186(42.7 \%)$ & \\
\hline
\end{tabular}

that they do not intent to receive a COVID-19 vaccine, 54 $(60.0 \%)$ attended the group lectures, 28 (31.1\%) arrived for on-site consultation and $15(16.7 \%)$ attended primary care visits to discuss their motives and concerns for vaccination (Table 1).

\section{Vaccination Rates}

Between January 3, and February 18, 2021, 459 (89.8\%) soldiers in the study population had been inoculated with the Pfizer-BioNTech COVID-19 vaccine. 454 (88.8\%) of soldiers had received both doses of the vaccine at the time of statistical analysis (Fig. 1).

\section{Changes in Vaccine Uptake}

All 421 soldiers who upon initial questioning were either intending to receive a vaccine or were unsure, accepted and received a vaccine. Of the 90 soldiers who did not intend to receive a vaccine, $38(42.2 \%)$ decided to receive a vaccine. $18(47.4 \%)$ of the 28 soldiers not intending to vaccinate and arriving for on-site physician consultation proceeded to vaccinate $(\mathrm{p}=0.004)$. We did not find statistically significant differences in vaccination rates among soldiers intending not to vaccinate, which attended group lectures or office visits (Table 2).

\section{Discussion}

To the best of our knowledge, this is the first study examining real-world uptake of a COVID-19 vaccine in a setting of unrestricted availability within a community, culminating in a vaccination rate of $89.8 \%$ in the military unit studied. Currently published data on COVID-19 vaccine uptake is predominantly based on hypothetical surveys of different populations, prior to vaccine emergency use authorization and availability [3, 6, 11-14]. While large-scale strategies to
Table 2 Attendance of interventions among soldiers not intending to vaccinate upon initial questioning, stratified according to vaccination status

\begin{tabular}{|c|c|c|c|c|}
\hline & \multicolumn{4}{|c|}{ COVID-19 Vaccination Status } \\
\hline & Vaccinated $(\mathrm{N}=38)$ & Unvaccinated $(\mathrm{N}=52)$ & Total $(\mathrm{N}=90)$ & $p$-value ${ }^{\mathrm{a}}$ \\
\hline \multicolumn{5}{|c|}{ Attendance of interventions among initially refusing soldiers $-\mathrm{N}(\%)$} \\
\hline Group lecture & $26(68.4 \%)$ & $28(53.8 \%)$ & $54(60.0 \%)$ & 0.119 \\
\hline On-site consultation & $18(47.4 \%)$ & $10(19.2 \%)$ & $28(31.1 \%)$ & $0.004^{*}$ \\
\hline Office-visit & $5(13.2 \%)$ & $10(19.2 \%)$ & $15(16.7 \%)$ & 0.320 \\
\hline
\end{tabular}


improve COVID-19 vaccine uptake have been discussed [4, $6,15]$, the value of informing individuals and groups at the community level cannot be understated [6]. Engagement of primary care physicians with their communities may alleviate concerns and reluctance for receiving a vaccine.

With the herd-immunity threshold for COVID-19 being estimated between 65 and $70 \%$ of a given population [16-18], public acceptance of a vaccine may stand as a significant hurdle to achieving this goal. In previous published surveys, the intent to vaccinate ranged between 34.8 and $85.8 \%[6,11-14,19,20]$, indicating that even upon unrestricted availability, achieving the herd immunity threshold via vaccination may be difficult. Burgress et al. [4] discussed different strategies to engage with communities on the topic of COVID-19 vaccine acceptance, calling for an active response from community leaders and healthcare workers. Policy makers should strive to provide physicians and other medical care providers with tools for community education, both online and in-person.

The communication efforts discussed in this study aimed to specifically target and dispel concerns which were identified in an anonymous survey within the same population prior to vaccine rollout. Researchers administering similar surveys have found that patients share comparable concerns regarding the safety profile, currently recognized and unforeseen side effects and the development and approval process of COVID-19 vaccines [3, 6, 11, 13, 14, 19]. The multi-layered approach implemented here, beginning with group sessions and culminating with primary care office visits, offers a community-based strategy to maximizing vaccine acceptance. The sequential approach of the interventions allowed for soldiers to initially discuss their concerns in groups composed of their platoon companions and later on in a more discrete and private manner whether on-site or in the clinic. Soldiers committed on vaccinating were also encouraged to attend the group sessions, aiming to boost positive attitudes and discussion on the vaccine within each platoon.

As of February 20, 2021, the overall vaccination rate within the unit was $89.8 \%$. We observed changes in soldier's willingness to receive a vaccine, with universal acceptance among soldiers initially unsure regarding the vaccine and a $42.2 \%$ acceptance rate among those initially refusing to vaccinate. Vaccine hesitancy is often complex and varies greatly across populations and different vaccines [21]. Strategies to improve public acceptance have been thoroughly discussed in the literature, but evaluating their effectiveness remains a great challenge [22]. The role of the primary care physician in addressing vaccine hesitancy has been discussed before, and presents some significant challenges including office time-constraints and feelings of frustration or mistrust between vaccine-hesitant families and physicians [2, 22, 23].

The limitations of this study must be emphasized, including the relatively small sample size and setting in a homogenous military unit with great access to COVID-19 vaccination and primary care interventions. Importantly, we cannot attribute causality of the voluntary interventions applied to any of the changes in vaccine acceptance. Soldiers' informed-decisions may have been swayed and influenced by an array of other factors such as socialmedia, public-outreach efforts, popular media, and attitudes within soldiers' families and home communities. Additionally, soldiers attending any of the interventions may have been more apt to vaccinate regardless.

However, our study highlights the potential value of vaccine accessibility within a young adult community in $a$ and offers several primary care strategies to address vaccine hesitancy. The findings and methodology in this study may be applied to other young adult communities such as higher education campuses, high-schools and military settings. Future studies should evaluate the effectiveness of such community interventions in a controlled manner across health networks and on the regional or national levels.

\section{Conclusions}

This study suggests that highly accessible vaccine sites and engagement of primary care physicians with their communities may aid patients in making informed decisions on uptake of COVID-19 vaccines, potentially increasing vaccination rates within their community.

Author Contributions TT: Conceptualization, Methodology, Formal Analysis, Data Curation, Writing — original draft. BC: Formal Analysis, Writing—review \& editing, IN: Methodology, Writing-review \& editing. YBM: Methodology, Data curation, Writing-review \& editing, Supervision.

Funding This research did not receive any specific grant or funding from any agency.

\section{Declarations}

Conflict of interest The authors declare the following financial interests or personal relationships which may be considered as potential competing interests: T.T. reports being an employee of Emedgene Technologies between 2017 and 2019, this affiliation has no connection or relevance to the currently submitted work. B.C, I.N and Y.B.M have no conflicts of interests to disclose.

Ethical Approval The IDF's institutional review board approved the use of the de-identified and aggregated data in this study. Vaccination and participation in any of the detailed interventions was entirely voluntary.

Consent to Participate All interventions were entirely voluntary. The IDF institutional review board waived the requirement for written informed consent. 


\section{References}

1. Danchin, M., Biezen, R., Manski-Nankervis, J.-A., Kaufman, J., \& Leask, J. (2020). Preparing the public for COVID-19 vaccines: How can general practitioners build vaccine confidence and optimise uptake for themselves and their patients? Australian Journal of General Practice, 49(10), 625-629. https://doi.org/10.31128/ AJGP-08-20-5559

2. Mohanty, S., Carroll-Scott, A., Wheeler, M., Davis-Hayes, C., Turchi, R., Feemster, K., et al. (2018). Vaccine hesitancy in pediatric primary care practices. Qualitative Health Research, 28(13), 2071-2080. https://doi.org/10.1177/1049732318782164

3. Dodd, R. H., Pickles, K., Nickel, B., Cvejic, E., Ayre, J., Batcup, C., et al. (2021). Concerns and motivations about COVID-19 vaccination. The Lancet Infectious Diseases, 21(2), 161-163. https:// doi.org/10.1016/S1473-3099(20)30926-9

4. Burgess, R. A., Osborne, R. H., Yongabi, K. A., Greenhalgh, T., Gurdasani, D., Kang, G., et al. (2021). The COVID-19 vaccines rush: Participatory community engagement matters more than ever. The Lancet, 397(10268), 8-10. https://doi.org/10.1016/ S0140-6736(20)32642-8

5. Feleszko, W., Lewulis, P., Czarnecki, A., \& Waszkiewicz, P. (2021). Flattening the curve of covid-19 vaccine rejection-An international overview. Vaccines, 9(1), 1-8. https://doi.org/10. 3390/vaccines9010044

6. Paul, E., Steptoe, A., \& Fancourt, D. (2020). Attitudes towards vaccines and intention to vaccinate against COVID-19: Implications for public health communications. The Lancet Regional Health-Europe. https://doi.org/10.1016/j.lanepe.2020.100012

7. Wilf-Miron, R., Myers, V., \& Saban, M. (2021). Incentivizing vaccination uptake: The "green pass" proposal in Israel. JAMA Journal of the American Medical Association. https://doi.org/10. 1001/jama.2021.4300

8. Mathieu, E., Ritchie, H., Ortiz-Ospina, E., Roser, M., Hasell, J., Appel, C., \& Giattino, C. (2021). A global database of COVID-19 vaccinations. medRxiv, (March), 2021.03.22.21254100. Retrieved April 24, 2020, from http://medrxiv.org/content/early/2021/03/26/ 2021.03.22.21254100.abstract

9. Talmy, T. (2021). Acceptance of and concerns about COVID19 vaccination: An online survey among Israel defense forces soldiers. Journal of Israeli Military Medicine. https://doi.org/10. 1371/journal.pone.0250555

10. Polack, F. P., Thomas, S. J., Kitchin, N., Absalon, J., Gurtman, A., Lockhart, S., et al. (2020). Safety and efficacy of the BNT162b2 mRNA Covid-19 Vaccine. The New England journal of medicine. https://doi.org/10.1056/NEJMoa2034577

11. Wang, K., Wong, E. L. Y., Ho, K. F., Cheung, A. W. L., Yau, P. S. Y., Dong, D., et al. (2021). Change of willingness to accept covid19 vaccine and reasons of vaccine hesitancy of working people at different waves of local epidemic in hong kong, china: Repeated cross-sectional surveys. Vaccines, 9(1), 1-15. https://doi.org/10. 3390/vaccines 9010062

12. Alley, S. J., Stanton, R., Browne, M., To, Q. G., Khalesi, S., Williams, S. L., et al. (2021). As the pandemic progresses, How does willingness to vaccinate against COVID-19 evolve? International Journal of Environmental Research and Public Health, 18(2), 797. https://doi.org/10.3390/ijerph18020797

13. Fisher, K. A., Bloomstone, S. J., Walder, J., Crawford, S., Fouayzi, H., \& Mazor, K. M. (2020). Attitudes toward a potential SARSCoV-2 vaccine : A Survey of U.S. adults. Annals of Internal Medicine, 173(12), 964-973. https://doi.org/10.7326/M20-3569

14. Dodd, R. H., Cvejic, E., Bonner, C., Pickles, K., McCaffery, K. J., Ayre, J., et al. (2020). Willingness to vaccinate against COVID-19 in Australia. The Lancet Infectious Diseases, 3099(20), 30559. https://doi.org/10.1016/S1473-3099(20)30559-4

15. Volpp, K. G., Loewenstein, G., \& Buttenheim, A. M. (2020). Behaviorally informed strategies for a National COVID-19 Vaccine Promotion Program. JAMA, 51(8), 1181-1192. https://doi. org/10.1001/jama.2020.24036

16. Sridhar, D., \& Gurdasani, D. (2021). Herd immunity by infection is not an option. Science, 371(6526), 230-231. https://doi.org/10. 1126/science.abf7921

17. Randolph, H. E., \& Barreiro, L. B. (2020). Herd immunity: Understanding COVID-19. Immunity, 52(5), 737-741. https://doi.org/ 10.1016/j.immuni.2020.04.012

18. Fontanet, A., \& Cauchemez, S. (2020). COVID-19 herd immunity: Where are we? Nature Reviews Immunology, 20(10), 583-584. https://doi.org/10.1038/s41577-020-00451-5

19. Lin, Y., Hu, Z., Zhao, Q., Alias, H., Danaee, M., \& Wong, L. P. (2020). Understanding COVID-19 vaccine demand and hesitancy: A nationwide online survey in China. PLOS Neglected Tropical Diseases, 14(12), e0008961. https://doi.org/10.1371/journal.pntd. 0008961

20. Neumann-Böhme, S., Varghese, N. E., Sabat, I., Barros, P. P., Brouwer, W., van Exel, J., et al. (2020). Once we have it, will we use it? A European survey on willingness to be vaccinated against COVID-19. European Journal of Health Economics, 21(7), 977982. https://doi.org/10.1007/s10198-020-01208-6

21. Larson, H. J., Jarrett, C., Eckersberger, E., Smith, D. M. D., \& Paterson, P. (2014). Understanding vaccine hesitancy around vaccines and vaccination from a global perspective: A systematic review of published literature, 2007-2012. Vaccine, 32(19), 2150-2159. https://doi.org/10.1016/j.vaccine.2014.01.081

22. Dubé, E., Gagnon, D., MacDonald, N. E., Eskola, J., Liang, X., Chaudhuri, M., et al. (2015). Strategies intended to address vaccine hesitancy: Review of published reviews. Vaccine, 33(34), 4191-4203. https://doi.org/10.1016/j.vaccine.2015.04.041

23. Dubé, E. (2017). Addressing vaccine hesitancy: The crucial role of healthcare providers. Clinical Microbiology and Infection, 23(5), 279-280. https://doi.org/10.1016/j.cmi.2016.11.007

Publisher's Note Springer Nature remains neutral with regard to jurisdictional claims in published maps and institutional affiliations. 